\title{
UMA ANÁLISE DA RELAÇÃO ENTRE O RETORNO DAS AÇÕES DO SETOR DE CONSTRUÇÃO CIVIL BRASILEIRO E INDICADORES MACROECONÔMICOS
}

\author{
Marcelo de Lima Balzana Filho ${ }^{1}$ \\ Ricardo Bordeaux-Rêgo ${ }^{2}$
}

\begin{abstract}
Resumo - Este trabalho teve como objetivo estudar os retornos das ações do setor de construção civil brasileiro em relação a indicadores macroeconômicos, visando descobrir se é possível relacionar os retornos das ações com os dos indicadores. A metodologia utilizada envolve o uso de testes de estacionariedade e análise de regressão multivariada, sendo aplicados a um universo de sete ações e oito indicadores econômicos. Os resultados apontam para a relevância do indicador de atividade Índice Bovespa no movimento das ações do setor imobiliário, influenciando todas as ações do estudo. Logo atrás daquele índice, vem a Taxa de Câmbio, que demonstrou poder explicativo em relação à maioria dos ativos do setor. A influência da inflação permanece duvidosa, pois, afetou significativamente somente três ações, e tendo seu real efeito de forma dúbia, influenciando algumas ações positivamente e outras negativamente.
\end{abstract}

Palavras-chave: Mercado de Ações, Ações da Construção Civil, Indicadores Macroeconômicos, Análise de Regressão.

\begin{abstract}
This study had the objective of studying the stock returns of the Brazilian construction industry in relation to macroeconomic indicators, in order to discover whether it is possible to relate the returns of the stocks with the indicators. The methodology involves the use of tests of stationarity and multivariate regression analysis, all been applied to a universe of seven stocks and eight economic indicators. The results point to the relevance of the index Ibovespa in the real estate industry, influencing every stock of the study. Just after that index, comes the Exchange Rate, which demonstrated explanatory power in relation to the majority of the sector's assets. Influence of Inflation could not be showed, as it significantly affected only three stocks, and affected some stocks positively and others negatively.
\end{abstract}

Keywords: Brazilian Stock Market, Brazilian Construction Sector, Macroeconomic Indicators, Regression Analysis.

\footnotetext{
${ }^{1}$ Dept $^{\circ}$ de Engenharia de Produção, Universidade Federal Fluminense. Campus Niterói.

${ }^{2}$ Dept $^{\circ}$ de Engenharia de Produção, Universidade Federal Fluminense. Campus Niterói.

ENGEVISTA, V. 16, n. 2, p.137-151, Junho 2014
} 


\section{Introdução}

As operações em bolsa de valores são consideradas pela sociedade brasileira como uma forma de auferir ganhos de capital superiores aqueles obtidos pela caderneta de poupança e pelos títulos públicos. Entretanto, é de conhecimento de todos que há também a possibilidade de altos prejuízos no mercado de ações para aqueles que não souberem aplicar o seu capital conhecendo o risco associado a este tipo de investimento. Esse risco, embutido nos investimentos, nada mais é do que a chance dos resultados reais serem diferentes dos esperados. Essa variabilidade, no movimento dos ativos, pode ser associada a índices de mercado ou quaisquer outras variáveis que se deseje, com intuito de criar um padrão para que se possa atribuir um grau de risco ao ativo. Não importa se as operações são de curto, médio ou longo prazo, sempre haverá um risco associado e entender isto pode ser o diferencial entre o investidor que sabe o que está fazendo e um que está apenas "jogando". Esse conhecimento do risco vem do vínculo que pode ser atribuído ao ativo em relação a alguma outra variável.

Usualmente se compara os retornos no mercado acionário com $\mathrm{o}$ índice de ações mais usado na economia local, essa comparação resulta no chamado risco sistêmico, que corresponde a variabilidade do retorno de uma ação em relação ao retorno do índice que representa o sistema no qual a ação esta inserida. Entender como diferentes ativos se comportam em relação às variáveis que o cercam é uma das formas de "mapear" o comportamento que o retorno desse ativo tem. No caso deste trabalho, serão avaliadas as ações do setor de construção civil brasileiro em relação a indicadores macroeconômicos externos as empresas, buscando montar equações usando os indicadores para explicar os retornos do setor imobiliário.

\subsection{Setor de Construção Civil}

Decidiu-se por analisar o setor imobiliário ou de construção civil devido à enorme atenção que está sendo despendida a este ramo nos últimos anos, que é resultado da grande alta nos preços dos imóveis, que pode ser consequência direta de uma demanda imobiliária bastante aquecida. Os números do setor, conforme dados do Relatório Setorial da Construção Civil feito pelo Departamento Intersindical de Estatística e Estudos Socioeconômicos (Dieese) em abril de 2011, tem mostrado que o mesmo está em expansão desde 2004, com aumento dos investimentos em obras de infraestrutura e na construção de imóveis habitacionais. Mesmo durante a crise, ocorrida em 2008, o PIB setorial cresceu $7,9 \%$, caindo em 2009, mas voltando a ter um desempenho de destaque no ano de 2010 , com alta de $11,6 \%$.

$\mathrm{O}$ aumento nos financiamentos de unidades imobiliárias nos últimos 3 anos tem ajudado o setor a ter o bom desempenho apresentado. Comparando 2010 com 2009, houve um crescimento de $73,09 \%$ nos valores contratados para financiamento habitacional através do Fundo de Garantia por Tempo de Serviço (FGTS), e um aumento de 65,20\% no financiamento habitacional com recursos advindos da poupança SBPE (Sistema Brasileiro de Poupança e Empréstimo). Totalizando, os créditos habitacionais tiveram um aporte de $\mathrm{R} \$ 83$ bilhões no ano de 2010, consequência também da queda dos juros e de programas como o "Minha Casa, Minha Vida" que facilitaram o acesso ao crédito para compradores com baixa renda.

Além desses resultados, há a expectativa de mais crescimento, no caso da indústria de construção pesada, decorrente das obras de infraestrutura para os preparativos da Copa do Mundo de 2014 e das Olimpíadas de 2016, que será sediada pela cidade do Rio de Janeiro. Há a necessidade de melhorias, com consequente uso do setor de construção, nas áreas de: infraestrutura de transportes, estádios, ginásios esportivos, aeroportos, readequação viária $\mathrm{e}$ saneamento. 


\subsection{Motivação e Objetivos}

Considerando sua importância na econômica brasileira, e sabendo que este ramo ainda é considerado novo em operações na Bolsa de Valores de São Paulo, com poucas ações alcançando representatividade no Índice Bovespa, surge a motivação deste trabalho que pode ser resumida em forma de pergunta:

Como estão se comportando as ações do setor de construção civil na bolsa de valores? Seria possível associar seus retornos a algum tipo de indicador macroeconômico, com poder estatístico?

O objetivo geral do trabalho é descobrir se há alguma relação entre os indicadores macroeconômicos e as ações do setor de construção civil brasileiro. A hipótese inicial é de que os indicadores econômicos influenciam os retornos acionários desse setor e que seja possível explicar estes retornos através das variações macroeconômicas. Sendo assim, serão feitos testes de correlação e análises de regressão para entender a relação contemporânea que há entre os ativos do setor e os indicadores. Após isso, o trabalho segue para o seu objetivo específico, o qual é montar equações de regressão que expliquem os retornos acionários do setor imobiliário por meio das mudanças das variáveis macroeconômicas.

\section{REVISÃO DA LITERATURA}

\subsection{Principais Mercado versus Macroeconômicos}

Teorias: Indicadores

Durante o século $\mathrm{XX}$, foram criadas algumas hipóteses que serviram para nortear a relação do mercado com os fatores econômicos, essas idéias se tornaram o senso comum do mercado internacional e dariam origem a muitos outros trabalhos no sentido de confirmar ou contestar suas afirmações. Tanto a confirmação quanto a contestação terminavam por gerar novas teorias para explicar as diferentes relações econômicas nos mercados do vários países do mundo.
As principais hipóteses criadas para explicar o funcionamento das bolsas durante $o$ século passado foram as seguintes:

1. Hipótese Fisheriana: Em Fisher (1930), é levantada a questão de que o investimento em ações seria um hedge para a inflação. Os retornos do mercado de ações seriam independentes das taxas de inflação, sendo influenciados por fatores reais da atividade econômica e funcionariam como hedge da inflação no longo prazo. (Adrangi et al., 2002, p. 63)

2. Hipótese do Proxy Effect: Fama (1981) utiliza os dados dos retornos acionários e da inflação do período pósguerra norte-americano (1953-1977) para mostrar que as ações não funcionam como hedge para a inflação. Na verdade, existe uma correlação negativa entre a bolsa e a inflação, que seria uma consequência da relação positiva entre as ações e a atividade econômica, que por sua vez tem uma correlação negativa com a inflação. $\mathrm{O}$ artigo ainda enfatiza que agrupando os dados anualmente, o efeito explicativo da inflação sobre as ações desaparece na presença da variável atividade real (Fama, 1981, p. 563).

3. Hipótese da Causalidade Reversa: Proposta por Geske and Roll (1983), pode ser considerado um complemento a teoria proposta por Fama (1981). A teoria apresentada é de que os preços das ações variam antes das outras variáveis, porque os investidores se apressam em prever as mudanças que irão ocorrer na economia e rapidamente descontam os resultados de seus cálculos nos ativos.

Diante dessas teorias propostas por esses três trabalhos, há uma série de artigos buscando comprovar, descartar ou ainda sugerir novas explicações para os diversos mercados financeiros ao redor do mundo. A seguir serão apresentadas as conclusões de alguns trabalhos que estudaram as relações entre os mercados acionários e indicadores macroeconômicos para diversas econômicas ao redor do mundo e também 
para o caso brasileiro, respectivamente nessa ordem.

\subsection{Mercados Internacionais}

Gultekin (1983) estuda as hipóteses de Fisher para 26 países (África do Sul, Alemanha, Austrália, Austria, Bélgica, Canadá, Dinamarca, Espanha, Estados Unidos, Filipinas, Finlândia, França, Holanda, Índia, Irlanda, Israel, Itália, Japão, Noruega, Nova Zelândia, Peru, Reino Unido, Singapura, Suécia, Suíça e Venezuela). O autor utilizou dados mensais no período de janeiro de 1947 até dezembro 1979. Foram criados modelos de regressão para medir o grau de relação dos mercados com suas respectivas inflações e os resultados da análise mostraram que os retornos acionários e a inflação não tem relação estatística significativa para a maioria das bolsas. $\mathrm{O}$ poder explicativo das regressões foi pequeno, sendo a correlação negativa entre as ações e a inflação detectada para 18 países. Dentre seis países, onde houve uma boa significância estatística, quatro tinham uma correlação negativa com a inflação. $\mathrm{O}$ destaque do estudo foi para o Reino Unido, sendo este o único país cuja relação dos retornos acionários e da inflação foi constatada como positiva e próxima de 1,0. O coeficiente Beta da regressão do Reino Unido foi de 0,705.

A conclusão do estudo foi de que a relação da inflação com as bolsas ao redor do mundo é predominantemente negativa.

O trabalho de Campbell (2004) realiza um estudo sobre a influência da inflação, do prêmio por risco e da volatilidade dos preços das ações sobre os dividendos. Esse estudo foi feito no mercado norte americano, no período de maio de 1927 até dezembro de 2002. As equações formuladas para o retorno dos dividendos conseguem explicar até $49 \%$, sendo sua equação com maior poder de explicação em função da inflação histórica e do prêmio por risco. As principais conclusões do artigo são que a inflação é positivamente correlacionada com o crescimento dos dividendos no longo prazo.

ENGEVISTA, V. 16, n. 2, p.137-151, Junho 2014
A pesquisa de Mahmood e Dinniah (2009) analisa o impacto das variáveis macroeconômicas no mercado de ações de seis países do pacífico asiático (Malásia, Coreia, Tailândia, Japão, Austrália e Hong Kong), que foram escolhidos por terem sido pouco afetados pela crise asiática. $\mathrm{O}$ estudo foi conduzido no período de janeiro de 1993 a dezembro de 2002 e foram utilizados como indicadores macroeconômicos a taxa de câmbio, inflação e atividade industrial. $\mathrm{O}$ objetivo inicial era tentar encontrar algum tipo de equilíbrio de longo prazo entre as variáveis e descobrir se existe alguma relação de causalidade no curto prazo. Os resultados indicam que há uma relação de equilíbrio no longo prazo entre todas as variáveis e as ações no Japão, Coreia, Hong Kong e Austrália. No curto prazo, detectou-se algum tipo de relação entre todas as variáveis e o mercado acionário para todos os países com exceção de Hong Kong, que demonstrou relação apenas com a taxa de câmbio, e Tailândia, que mostrou alguma interação somente com a atividade industrial.

O trabalho de Singh (2010) que aborda as relações entre o mercado da Índia, a inflação, produção industrial e a taxa de câmbio, usando dados mensais do período de abril de 1995 a março de 2009. São realizados testes de correlação e causalidade de Granger entre as quatro variáveis, o objetivo principal é descobrir qual o grau de causalidade entre elas, seja unilateral ou bilateral. A análise dos resultados se mostrou bastante interessante, constatou-se que todas as variáveis possuem correlações positivas altas, com exceção da taxa de câmbio que possui uma correlação positiva baixa com as outras variáveis. A causalidade de Granger mostrou que o índice de ações causa variações na atividade industrial e na inflação, sendo que somente a atividade industrial tem o mesmo efeito no índice. A taxa de câmbio não demonstrou causar e nem sofrer causalidade por nenhuma das variáveis, sugerindo que suas variações devem ser explicadas por outros indicadores 
externos ao estudo. A conclusão do artigo é de que o mercado de ações indiano ainda é pouco desenvolvido, com poucas pessoas investindo, e que desse modo a relação deste com as variáveis econômicas ainda não seja consistente e madura suficiente para se extrair relações conclusivas a respeito da economia da Índia.

A utilização de regressões também foi observada no trabalho de Dasgupta et. al. (2011), que concluiu que os retornos negativos da constante negociação institucional de ações não é explicada pelos retornos passados, se concentra nas ações de menor valor e apresenta maior intensidade nas empresas com maior concentração de propriedade institucional.

Em seu trabalho sobre problemas de Agência em empresas aéreas de capital aberto, Edgerton (2012) utilizou a regressão para concluir que as empresas de propriedade de fundos de Private Equity têm em média, frotas quarenta por cento menores do que as outras de capital aberto. O trabalho de Bergh e Gibbons (2011) estudou a reação das ações ao anúncio da contratação de consultorias de gestão. Os autores utilizaram a regressão para concluir que, para a amostra de 118 empresas que anunciaram publicamente a contratação de consultorias, o mercado reagiu favoravelmente. A regressão apontou que a reação tendia a ser maior nas empresas com maior lucratividade.

Hendershott et al (2011) realizaram estudo para inferir a relação entre a utilização de algoritmos e o aumento da liquidez. Os autores se valeram da regressão para verificar que o aumento da negociação automática a partir de 2003 está relacionado à utilização de algoritmos de negociação. O trabalho de Chi Ming Ho (2011) emprega a regressão ao relacionar a autoconfiança dos investidores de Taiwan e sua disposição de investir em mercados em baixa. $\mathrm{O}$ autor também conclui que os investidores confiantes preferem investir em ações de empresas com pequena capitalização.
O trabalho recente de Ferri e Maber (2013) utilizou regressões para avaliar o impacto de uma nova regulamentação sobre transparência no pagamentos a executivos na Grã-Bretanha. Os resultados apontam para uma reação positiva das cotações das ações de empresas que apresentavam poucas penalidades ao baixo desempenho. Devido à transparência exigida pela regulamentação, as empresas britânicas eliminaram as práticas consideradas premiação a maus resultados.

A seguir, a revisão de trabalhos focados no mercado brasileiro.

\subsection{Mercado Brasileiro}

Adrangi et al. (2002) estudam o mercado de ações brasileiro, no período de janeiro de 1986 a julho de 1997, com o objetivo de testar a hipótese do proxy effect para uma econômica emergente, a qual era, e ainda é, considerada a maior econômica da América Latina. Os resultados apontaram para uma aceitação da hipótese de Fama no longo prazo, pois embora tenha sido constatado que o mercado acionário brasileiro possui uma relação negativa com a inflação, essa relação continuava existindo mesmo após retirar do modelo os efeitos da correlação negativa entre a atividade real e a inflação. Descobriu-se que os retornos acionários, a inflação e a atividade econômica possuem uma curva de equilíbrio apenas no longo prazo, não sendo este equilíbrio detectado no curto prazo. A conclusão é de que no caso do Brasil o proxy effect seria observado apenas em períodos mais longos.

Em Grôppo (2004), é investigada a existência de possíveis relações causais entre um conjunto de variáveis macroeconômicas e o Índice Bovespa, o qual foi escolhido neste trabalho para representar o mercado de ações. $\mathrm{O}$ período do estudo compreende janeiro de 1995 a dezembro de 2003, e a técnica econométrica de modelos de autoregressão vetorial (VAR) foi usada para medir as relações do Ibovespa com a taxa de câmbio, o preço do barril de petróleo no mercado internacional, a taxa de juros 
de curto prazo deflacionada e o índice de produção industrial. Após a análise das relações de longo prazo do mercado com esses quatro indicadores, descartou-se a hipótese de Fisher e Fama, pois os resultados apontaram uma correlação negativa de longo prazo do índice de ações com todas as quatro variáveis independentes. Isso significa que $o$ Ibovespa responde negativamente a impulsos ou choques que ocorram na outras variáveis, tendo sido medido por Grôppo o nível de resposta da variável dependente para um aumento de $10 \%$ nas variáveis independentes: aumento na SELIC, queda de 12,6\%; aumento na taxa de câmbio, queda de 1,3\%; aumento no preço do barril de petróleo, queda de $15,14 \%$ no segundo mês. O autor conclui o trabalho destacando que a taxa básica de juros da economia é a variável com maior ligação com o mercado de ações, sugerindo que isso se deve aos investidores enxergarem a renda fixa como um substituto para os investimentos em renda variável.

A pesquisa de Castro (2009) teve como principal objetivo verificar se o mercado brasileiro possuía a mesma relação negativa entre a inflação e retornos acionários, a qual foi observada por diversos artigos para as economias internacionais. A base estudada compreendia os retornos mensais do IPCA, IGP-M, taxa de câmbio, commodities, índices setoriais e o Índice Bovespa, desde janeiro de 2000 até setembro de 2008. No começo, Castro testou o IPCA como variável independente frente ao Ibovespa $\mathrm{e}$ concluiu que não havia poder explicativo, testou então o IGP-M e obteve os mesmos resultados. Em seguida, decompôs o IPCA nas componentes antecipada e não antecipada e ainda assim não encontrou resultados diferentes, passando para a análise das commodities e da taxa de câmbio, onde encontrou significância no modelo. Há ainda, no final da pesquisa, uma análise idêntica para os diversos setores econômicos do mercado de ações, incluindo o setor de construção civil, cabendo ressaltar aqui que não foram encontradas relações significativas entre as variáveis estudadas e este setor.

O artigo de Bertella et al. (2009) busca relacionar o mercado de ações, de 1995 a 2007, com indicadores macroeconômicos, separando estes em internos e externos ao Brasil. Desse modo, são estudados como indicadores internos: índice de produção industrial, índice de inflação, taxa de juros real, risco de crédito doméstico e taxa de câmbio real. Os indicadores externos que fazem parte do estudo são: Standard e Poor`s 500, taxa de juros americana e o preço do barril de petróleo. Utilizando diversas metodologias econométricas, encontraram-se relações positivas de longo prazo entre o Ibovespa com a bolsa americana e o preço do petróleo, e relações negativas com a taxa de juros americana e a taxa de câmbio real, as outras variáveis não tiverem significância considerável. Esses resultados confirmam alguns encontrados por trabalhos anteriores, como a taxa de câmbio negativa, e divergem em outros pontos, como a relação do preço do petróleo negativa encontrada por Grôppo (2004). Os autores terminam o artigo ressaltando o peso que os indicadores externos ao Brasil tem em seu mercado acionário, mas destacam que de todos as variáveis, o dólar foi aquela com maior grau de resposta significativa.

\section{METODOLOGIA}

\subsection{Análise de Regressão}

Será utilizada a análise de regressão linear multivariada para associar os retornos das ações do setor de construção civil com os indicadores macroeconômicos. A regressão multivariada é um método usado para relacionar uma variável que será chamada de dependente ou condicionada com outras variáveis que são denominadas independentes ou condicionais. É dita linear porque tem como objetivo montar uma equação com variáveis de primeiro grau, que sirva para prever os valores que a variável explicada terá, dado os valores 
das variáveis explicativas. A equação de regressão multivariada tem o seguinte formato:

$$
Y_{i}=\alpha+\beta_{1} \mathrm{X}_{1}+\beta_{2} \mathrm{X}_{2}+\ldots+\beta_{n} \mathrm{X}_{n}+\varepsilon_{i}
$$

Onde $Y_{i}$ é a variável dependente; $\alpha$ é o valor base de $Y_{i}$, correspondendo ao intercepto da equação; $\beta_{i}$ é o coeficiente linear da i-ésima variável; $\mathrm{X}_{i}$ representa a i-ésima variável explicativa e $\varepsilon_{i}=Y_{i}-\hat{Y}_{i}$, representa o erro da medição e todos os fatores residuais da equação.

Para que a análise de regressão seja realizada corretamente, faz-se necessário atentar para os pressupostos da linearidade, normalidade dos resíduos, homocedasticidade, independência dos resíduos e inexistência de colinearidade. Quase todas as condições podem ser verificadas analisando o gráfico dos resíduos das regressões, com exceção da colinearidade, que é verificada observando se as correlações das variáveis dependentes é inferior a 0,7. Ademais, o modo de medir se uma equação de regressão está bem ajustada aos dados pode ser feita através do coeficiente de determinação $R^{2}$. Essa estatística serve para medir o quanto da variabilidade total das observações da série pode ser explicada pela reta de regressão traçada, o que significa que quanto maior o valor de $R^{2}$, melhor será o ajuste do modelo. O coeficiente $R^{2}$ segue a seguinte fórmula:

$$
R^{2}=\frac{S Q R}{S Q T}=1-\frac{S Q E}{S Q T}
$$

Onde SQRé a soma dos quadrados devido a regressão ou $\sum\left(\hat{y}_{i}-\bar{y}\right)^{2}$; $S Q E$ é a soma dos quadrados dos resíduos ou $\sum \hat{e}_{i}^{2} ; S Q T$ é a soma dos quadrados totais ou $\sum\left(y_{i}-\bar{y}\right)^{2}$.

\subsection{Teste de Raiz Unitária de Dickey-Fuller Aumentado}

Uma série é chamada de estacionária se possui a mesma média e variância para todos os seus valores ao longo do tempo. A condição da estacionariedade é um requisito importante ao relacionar variáveis econômicas, pois quando uma série econômica é não-estacionária qualquer regressão realizada com outra variável é considerada espúria, mesmo que essa regressão mostre alta significância estatística. Para descobrir se uma série é estacionária, este trabalho utilizará o teste de raiz unitária de Dickey-Fuller Aumentado (ADF), que é derivado do teste de Dickey-Fuller (DF), apresentado em Dickey e Fuller (1979), e se baseia em testar os seguintes processos autoregressivos:

$$
\begin{aligned}
& Y_{t}=\rho Y_{t-1}+\sum_{i=1}^{p-1} \delta_{i} \Delta Y_{t-i}+\varepsilon_{t} \\
& Y_{t}=\alpha+\rho Y_{t-1}+\sum_{i=1}^{p-1} \delta_{i} \Delta Y_{t-i}+\varepsilon_{t} \\
& Y_{t}=\alpha+\beta t+\rho Y_{t-1}+\sum_{i=1}^{p-1} \delta_{i} \Delta Y_{t-i}+\varepsilon_{t}
\end{aligned}
$$

Onde $Y_{t}$ é o valor da série no tempo t; $\alpha$ é o intercepto; $\beta t$ é o termo que representa a tendência determinística;

$$
\Delta Y_{t-i}=Y_{t-i}-Y_{t-i-1} ; \sum_{i=1}^{p-1} \delta_{i} \Delta Y_{t-i} \text { testa a }
$$

possibilidade de $Y_{t}$ ser influenciado por observações mais defasadas de ordem $Y_{t-i}$, sendo i $>1$, e $\varepsilon_{t}$ é uma sequência de variáveis aleatórias independentes e identicamente distribuídas, com distribuição $\quad N\left(0, \sigma^{2}\right) \quad$ e $\quad$ sem autocorrelação.

Se $/ \rho /<1$, então a série irá convergir para uma série estacionária no infinito, porém se o valor de $/ \rho /=1$, a série será não estacionária e sua variância será igual a $t \sigma^{2}$, sendo $Y_{t}$ mais conhecida, nesse caso específico, como um passeio aleatório. Em virtude disso, o objetivo do teste ADF é testar a hipótese nula de que a série é não estacionária $(/ \rho /=1)$, contra a hipótese alternativa de que a série é estacionária $(/ \rho /<1)$. Os valores críticos usados para rejeitar a 
hipótese de raiz unitária são extraídos da distribuição $\tau$ que foi tabulada por Fuller (Margarido e Anefalos, 1999, p. 22). A distribuição $\tau$ é usada no caso da equação (3), mas se o modelo possuir intercepto usa-se a equação (4) e a estatística $\tau_{\mu}$, e se o modelo possuir intercepto $\mathrm{e}$ tendência, equação (5) e a estatística $\tau_{\tau}$.

O número de defasagens considerado ideal para eliminar a autocorrelação dos resíduos será calculado pelo Critério de Informação de Akaike (AIC), que utiliza a seguinte fórmula para um número reduzido de observações (quando $n / k<40$ ):

$$
A I C=n \ln \left(\frac{S Q E}{n}\right)+2 k+\frac{2 k(k+1)}{n-k-1}
$$

Onde SQE é a soma dos quadrados dos resíduos; $k$ é o número de parâmetros e $n$ é o número de observações.

\subsection{Base de Dados}

Primeiramente, as cotações das ações foram recolhidas no site oficial da Bolsa de Valores de São Paulo e em seguida foram selecionadas as ações do setor imobiliário. O critério para essa seleção é feito com base nos ativos que compõem o Índice Imobiliário (IMOB) e também o Índice Bovespa (IBOV). Foi decidido utilizar apenas os ativos que estivessem na interseção dos dois índices para garantir a liquidez, que é essencial para que se consiga medir as possíveis influências dos indicadores nos retornos das ações escolhidas. Desse modo, foram selecionadas sete ações: segundo Ross (2008), para a manutenção das atividades de uma empresa, esta terá que realizar investimentos em capital de giro líquido além dos investimentos em ativos a longo prazo. Esses valores considerar-se-ão saídas de caixa ao longo período de atividade da companhia.

A maior parte dos indicadores econômicos foi escolhida com base nos diversos trabalhos da literatura estudada.
A inflação será representada pelo INCC, IPCA e IGP-M, o primeiro foi escolhido por representar diretamente o aumento dos custos referentes à construção civil, o segundo é o índice mais difundido para medir a inflação da econômica brasileira e o terceiro é o índice utilizado para corrigir contratos de aluguel. O IPCA é calculado pelo Instituto Brasileiro de Geografia e Estatística (IBGE), enquanto o INCC e o IGP-M são calculados pela Fundação Getúlio Vargas (FGV), a base de dados mensal dos três índices foi extraída do Portal Brasil (www.portalbrasil.net/indices).

Em relação aos indicadores financeiros, a Taxa Selic, que será representada pelos Certificados de Depósito Interbancário (CDI), teve seus dados mensais também retirados do Portal Brasil. A taxa de câmbio no formato (R\$/US\$) foi extraída diretamente do site do Banco Central (www.bcb.gov.br).

Por último, os índices de atividade: PIB, Ibovespa e Taxa de Desocupação. O PIB está representando o crescimento da atividade econômica do país e seu banco de dados mensal foi retirado do IPEADATA (www.ipeadata.gov.br). As cotações de fechamento do Índice Bovespa foram extraídas do site Bolsapt (www.bolsapt.com). A taxa de desocupação mensal foi obtida no banco de dados do IBGE (www.ibge.gov.br).

\section{Dados \\ 3.4. Análise Descritiva dos}

Em virtude das datas da primeira oferta pública das ações escolhidas como parte da pesquisa, conforme tabela 1 , foi decidido realizar a análise no período de 01 de agosto de 2007 até 31 de dezembro de 2011. O estudo será feito utilizando os retornos mensais de todas as ações e indicadores econômicos selecionados, contando com um total de 53 observações. 
Tabela 1: Informações das ações analisadas

\begin{tabular}{|cccccc|}
\hline Código & Ação & Tipo & $\mathbf{1}^{\circ}$ Oferta & Part. IMOB (\%) & Part. IBOV (\%) \\
\hline BISA3 & BROOKFIELD & ON & $23 / 10 / 2006$ & $2,74 \%$ & $0,82 \%$ \\
\hline BRML3 & BR MALLS PAR & ON & $05 / 04 / 2007$ & $19,45 \%$ & $1,00 \%$ \\
\hdashline CYRE3 & CYRELA REALT & ON & $01 / 07 / 2005$ & $9,48 \%$ & $1,92 \%$ \\
\hline GFSA3 & GAFISA & ON & $17 / 02 / 2006$ & $4,35 \%$ & $1,81 \%$ \\
\hline MRVE3 & MRV & ON & $23 / 07 / 2007$ & $8,93 \%$ & $1,89 \%$ \\
\hline PDGR3 & PDG REALT & ON & $26 / 01 / 2007$ & $15,62 \%$ & $2,99 \%$ \\
\hline RSID3 & ROSSI RESID & ON & $05 / 01 / 2005$ & $3,45 \%$ & $1,35 \%$ \\
\hline TOTAL & & & & $64,02 \%$ & $11,78 \%$ \\
\hline
\end{tabular}

Fonte: www.bmfbovespa.com.br em 19/03/2012

A tabela 2 contém um resumo das principais estatísticas das variáveis que serão usadas no estudo:

Tabela 2: Estatísticas descritivas dos retornos mensais

\begin{tabular}{|c|c|c|c|c|c|c|}
\hline Variável & $\begin{array}{c}\text { Média } \\
\text { Aritmética }\end{array}$ & Des vio Padrão & Mínimo & Máximo & Amplitude & Rent. Acumulada \\
\hline \multicolumn{7}{|l|}{ Ações } \\
\hline BISA3 & $-0,41 \%$ & $19,31 \%$ & $-32,31 \%$ & $92,31 \%$ & $124,62 \%$ & $-65,21 \%$ \\
\hline BRML3 & $1,62 \%$ & $12,28 \%$ & $-30,43 \%$ & $30,20 \%$ & $60,63 \%$ & $57,57 \%$ \\
\hline CYRE3 & $0,69 \%$ & $17,13 \%$ & $-45,64 \%$ & $48,26 \%$ & $93,90 \%$ & $-32,70 \%$ \\
\hline GFSA 3 & $-0,77 \%$ & $18,52 \%$ & $-42,60 \%$ & $62,92 \%$ & $105,52 \%$ & $-72,52 \%$ \\
\hline MRVE3 & $1,52 \%$ & $16,98 \%$ & $-42,36 \%$ & $54,55 \%$ & $96,91 \%$ & $3,88 \%$ \\
\hline PDGR3 & $1,24 \%$ & $15,35 \%$ & $-31,48 \%$ & $59,20 \%$ & $90,68 \%$ & $8,76 \%$ \\
\hline RSID3 & $-0,10 \%$ & $21,31 \%$ & $-46,00 \%$ & $104,05 \%$ & $150,05 \%$ & $-66,53 \%$ \\
\hline \multicolumn{7}{|c|}{ Indicadores Financeiros } \\
\hline CDI & $0,87 \%$ & $0,13 \%$ & $0,59 \%$ & $1,17 \%$ & $0,58 \%$ & $57,99 \%$ \\
\hline Dolar & $0,13 \%$ & $5,31 \%$ & $-9,43 \%$ & $17,13 \%$ & $26,56 \%$ & $-0,09 \%$ \\
\hline \multicolumn{7}{|c|}{ Indicadores de Preços } \\
\hline INCC & $0,59 \%$ & $0,59 \%$ & $-0,25 \%$ & $2,94 \%$ & $3,19 \%$ & $36,79 \%$ \\
\hline IPCA & $0,46 \%$ & $0,22 \%$ & $0,00 \%$ & $0,83 \%$ & $0,83 \%$ & $27,20 \%$ \\
\hline IGPM & $0,55 \%$ & $0,63 \%$ & $-0,74 \%$ & $1,98 \%$ & $2,72 \%$ & $33,72 \%$ \\
\hline \multicolumn{7}{|c|}{ Indicadores de Atividade } \\
\hline IBOV & $0,36 \%$ & $7,30 \%$ & $-24,80 \%$ & $15,55 \%$ & $40,35 \%$ & $4,75 \%$ \\
\hline PIB & $0,98 \%$ & $3,68 \%$ & $-9,66 \%$ & $6,99 \%$ & $16,65 \%$ & $61,60 \%$ \\
\hline Txdes & $-1,15 \%$ & $5,99 \%$ & $-10,53 \%$ & $20,59 \%$ & $31,12 \%$ & $-50,53 \%$ \\
\hline
\end{tabular}

Fonte: Elaborado pelos autores

Pode-se notar que, dentre as ações, BRML3 teve a maior média dos retornos mensais, o menor desvio padrão e também a maior rentabilidade acumulada no período, mas ainda assim, por muito pouco, não foi capaz de superar o $\mathrm{CDI}$, o qual teve uma rentabilidade acumulada de aproximadamente 58\%. Percebe-se que o desvio padrão das ações fica entre $12,28 \%$ (BRML3) e $21,31 \%$ (RSID3), enquanto o IBOV tem um desvio padrão no valor de $7,30 \%$, indicando que as ações possuem um risco maior que o índice do mercado. Segue o cálculo do coeficiente de Correlação de Pearson com $\mathrm{o}$ teste $\mathrm{t}$ associado à hipótese nula dos coeficientes serem iguais a zero, conforme a Tabela 3. 
Tabela 3: Coeficiente de Correlação de Pearson

\begin{tabular}{|c|c|c|c|c|c|c|c|c|}
\hline Variável & CDI & Dolar & INCC & IPCA & IGPM & IBOV & PIB & Txdes \\
\hline \multicolumn{9}{|l|}{ Ações } \\
\hline \multirow{2}{*}{ BISA3 } & $(0,380)$ & $(0,568)$ & $(0,193)$ & $(0,093)$ & $(0,213)$ & 0,641 & $(0,001)$ & $(0,105)$ \\
\hline & $0,005 * * *$ & $0,000 * * *$ & 0,166 & 0,507 & 0,126 & $0,000 * * *$ & 0,994 & 0,455 \\
\hline \multirow{2}{*}{ BRML3 } & $(0,227)$ & $(0,283)$ & $(0,160)$ & $(0,070)$ & $(0,255)$ & 0,604 & 0,011 & 0,102 \\
\hline & 0,103 & $0,04 * *$ & 0,253 & 0,616 & $0,066^{*}$ & $0,000 * * *$ & 0,936 & 0,469 \\
\hline \multirow{2}{*}{ CYRE3 } & $(0,224)$ & $(0,616)$ & $(0,184)$ & $(0,304)$ & $(0,325)$ & 0,770 & 0,088 & $(0,072)$ \\
\hline & 0,107 & $0,000 * * *$ & 0,186 & $0,027 * *$ & $0,018^{* *}$ & $0,000 * * *$ & 0,531 & 0,607 \\
\hline \multirow{2}{*}{ GFSA 3} & $(0,219)$ & $(0,550)$ & $(0,262)$ & $(0,222)$ & $(0,267)$ & 0,684 & $(0,008)$ & 0,002 . \\
\hline & 0,115 & $0,000 * * *$ & $0,058^{*}$ & 0,110 & $0,053^{*}$ & $0,000 * * *$ & 0,954 & 0,986 \\
\hline \multirow{2}{*}{ MRVE3 } & $(0,279)$ & $(0,697)$ & $(0,143)$ & $(0,128)$ & $(0,260)$ & 0,765 & 0,229 & $(0,006)$ \\
\hline & $0,043^{* *}$ & $0,000 * * *$ & 0,306 & 0,363 & $0,06^{*}$ & $0,000 * * *$ & $0,1^{*}$ & 0,966 \\
\hline \multirow{2}{*}{ PDGR3 } & $(0,191)$ & $(0,644)$ & $(0,187)$ & $(0,104)$ & $(0,210)$ & 0,736 & 0,188 & 0,002 \\
\hline & 0,171 & $0,000^{*} * *$ & 0,181 & 0,457 & 0,130 & $0,000 * * *$ & 0,177 & 0,986 \\
\hline \multirow{2}{*}{ RSID3 } & $(0,240)$ & $(0,614)$ & $(0,231)$ & $(0,137)$ & $(0,240)$ & 0,681 & 0,146 . & 0,014 \\
\hline & $0,084^{*}$ & $0,000 * * *$ & $0,096^{*}$ & 0,329 & $0,083^{*}$ & $0,000 * * *$ & 0,297 & 0,922 \\
\hline \multicolumn{9}{|c|}{ Indicadores Financeiros } \\
\hline \multirow{2}{*}{ CDI } & 1,000 & 0,362 & 0,143 & $(0,119)$ & $(0,029)$ & $(0,386)$ & $(0,002)$ & 0,077 \\
\hline & $0,000 * * *$ & $0,008 * * *$ & 0,306 & 0,398 & 0,834 & $0,004 * * *$ & 0,987 & 0,585 \\
\hline \multirow{2}{*}{ Dolar } & 0,362 & 1,000 & 0,017 & 0,005 & $(0,025)$ & $(0,675)$ & $(0,217)$ & 0,049 \\
\hline & $0,008 * * *$ & $0,000 * * *$ & 0,906 & 0,970 & 0,857 & $0,000 * * *$ & 0,119 & 0,728 \\
\hline \multicolumn{9}{|c|}{ Indicadores de Preços } \\
\hline \multirow{2}{*}{ INCC } & 0,143 & 0,017 & 1,000 & 0,208 & 0,377 & $(0,260)$ & 0,281 & $(0,050)$ \\
\hline & 0,306 & 0,906 & $0,000 * * *$ & 0,134 & $0,005^{* * *}$ & $0,061^{*}$ & $0,041 * *$ & 0,724 \\
\hline \multirow{2}{*}{ IPCA } & $(0,119)$ & 0,005 & 0,208 & 1,000 & 0,513 & $(0,117)$ & $(0,083)$ & 0,209 \\
\hline & 0,398 & 0,970 & 0,134 & $0,000 * * *$ & $0,000 * * *$ & 0,405 & 0,554 & 0,133 \\
\hline \multirow{2}{*}{ IGPM } & $(0,029)$ & $(0,025)$ & 0,377 & 0,513 & 1,000 & $(0,232)$ & 0,063 & $(0,065)$ \\
\hline & 0,834 & 0,857 & $0,005^{* * *}$ & $0,000 * * *$ & $0,000 * * *$ & $0,094^{*}$ & 0,655 & 0,641 \\
\hline \multicolumn{9}{|c|}{ Indicadores de Atividade } \\
\hline \multirow{2}{*}{ IBOV } & $(0,386)$ & $(0,675)$ & $(0,260)$ & $(0,117)$ & $(0,232)$ & 1,000 & 0,093 & $(0,044)$ \\
\hline & $0,004 * * *$ & $0,000 * * *$ & $0,061^{*}$ & 0,405 & $0,094^{*}$ & $0,000 * * *$ & 0,509 & 0,755 \\
\hline \multirow{2}{*}{ PIB } & $(0,002)$ & $(0,217)$ & 0,281 & $(0,083)$ & 0,063 & 0,093 & 1,000 & $(0,149)$ \\
\hline & 0,987 & 0,119 & $0,041 * *$ & 0,554 & 0,655 & 0,509 & $0,000 * * *$ & 0,288 \\
\hline \multirow{2}{*}{ Txdes } & 0,077 & 0,049 & $(0,050)$ & 0,209 & $(0,065)$ & $(0,044)$ & $(0,149)$ & 1,000 \\
\hline & 0,585 & 0,728 & 0,724 & 0,133 & 0,641 & 0,755 & 0,288 & $0,000 * * *$ \\
\hline
\end{tabular}

Fonte: Elaborado pelos autores

\section{ANÁLISE DOS RESULTADOS}

Primeiramente, foi realizado o teste de Dickey-Fuller Aumentado (ADF) para identificar se as séries deste estudo são estacionárias e para isso foi utilizado o software estatístico SAS. Testou-se a hipótese nula de "não estacionariedade" das séries para os três modelos do teste ADF e a hipótese de estacionariedade foi rejeitada para todas as séries com significância estatística de $5 \%$ e $1 \%$, em nível, com exceção do CDI e do IPCA. Esses somente se tornaram estacionários depois da primeira diferenciação. Após a verificação e confirmação da estacionariedade das séries temporais, é possível realizar a análise de regressão multivariada das ações em relação aos indicadores econômicos. 


\subsection{Análise de Regressão Multivariada}

Foi realizada a análise de regressão multivariada dos retornos das sete ações com os oito indicadores macroeconômicos, utilizando o software SPSS 15.0. Para se obter o melhor modelo possível, foi escolhido que o programa executasse a regressão por meio do método backward, que significa realizar a regressão com todos os indicadores e ir removendo aqueles que tiverem uma significância acima de $10 \%$. É removido no máximo um indicador por regressão, aquele com a maior significância, e uma nova regressão é modelada, de modo que no final sobrem apenas variáveis independentes com significância menor do que $10 \%$. Acredita-se que por meio deste método todas as variáveis condicionais terão a mesma chance de mostrar seu poder explicativo em relação às ações. Um detalhe é que alguma variável excluída nos primeiros passos possa ter um poder explicativo considerado elevado, mas foi retirada do modelo porque seu grau de explicação é fraco na presença de outras variáveis, o que ocorre normalmente quando duas variáveis independentes medem o mesmo efeito na variabilidade da regressão.

Tabela 4: Modelos de regressão multivariados

\begin{tabular}{|c|c|c|c|c|c|c|c|c|c|c|}
\hline Ações & Constante & CDI & Dolar & INCC & IPCA & IGPM & IBOV & PIB & Txdes & $\mathrm{R}^{2}$ Ajustado \\
\hline \multirow{2}{*}{ BISA3 } & $-0,007$ & - & $-0,905$ & - & - & - & 1,252 & - & - & \multirow{2}{*}{0,422} \\
\hline & 0,718 & - & $0,088^{*}$ & - & - & - & $0,002 * * *$ & - & - & \\
\hline \multirow{2}{*}{ BRML3 } & 0,013 & - & - & - & - & - & 1,017 & - & - & \multirow{2}{*}{0,353} \\
\hline & 0,358 & - & - & - & - & - & $0,000 * * *$ & - & - & \\
\hline \multirow{2}{*}{ CYRE3 } & 0,001 & - & - & - & $-18,345$ & - & 1,832 & - & - & \multirow{2}{*}{0,620} \\
\hline & 0,927 & - & - & - & $0,020 * *$ & - & $0,000 * * *$ & - & - & \\
\hline \multirow{2}{*}{ GFSA3 } & $-0,014$ & - & - & - & - & - & 1,737 & - & - & \multirow{2}{*}{0,458} \\
\hline & 0,463 & - & - & - & - & - & $0,000 * * *$ & - & - & \\
\hline \multirow{2}{*}{ MRVE3 } & 0,037 & - & $-1,236$ & - & - & $-4,370$ & 1,086 & - & - & \multirow{2}{*}{0,648} \\
\hline & $0,062 *$ & - & $0,002 * * *$ & - & - & $0,071^{*}$ & $0,000 * * *$ & - & - & \\
\hline \multirow{2}{*}{ PDGR3 } & 0,009 & - & $-0,781$ & - & - & - & 1,164 & - & - & \multirow{2}{*}{0,565} \\
\hline & 0,508 & - & $0,034 * *$ & - & - & - & $0,000 * * *$ & - & - & \\
\hline \multirow{2}{*}{ RSID3 } & 0,036 & - & $-1,668$ & - & 20,347 & $-7,147$ & 1,001 & - & - & \multirow{2}{*}{0,519} \\
\hline & 0,223 & - & $0,005 * * *$ & - & $0,087^{*}$ & $0,057^{*}$ & $0,023 * *$ & - & - & \\
\hline
\end{tabular}

*** Significância de $1 \%$

** Significância de 5\%

* Significância de 10\%

Observando a tabela 4, percebe-se que o IBOV é a única variável independente que parece ter algum relação com todas as ações que compõem o estudo, tendo coeficiente positivo e maior que 1,000 , e significância menor que $1 \%$ em seis ações, todos os ativos parecem acompanhar concomitantemente o IBOV de modo mais volátil. Destacando que CYRE3 tem o maior coeficiente, 1,832, e RSID3 tem o menor coeficiente, da ordem de 1,001.

A Taxa de Câmbio se mostra influente para quatro ações, tendo significância de $1 \%, 5 \%$ e $10 \%$ dependendo do ativo. Seu valor é negativo para todos que influencia, variando do menor valor para RSID3, 1,668 , e o maior valor para PDGR3, 0,781 .

Os resultados para a inflação são um tanto controversos, já que o IPCA se mostra positivamente relacionado a uma ação, mas negativamente relacionado a outra. Na RSID3, onde o IPCA tem beta positivo, há um "atenuante", onde o IPGM parece explicar algum efeito não capturado pelo IPCA. Cabe ressaltar que os coeficientes do IPCA são consideravelmente maiores em módulo, porque esta série teve seus retornos 
diferenciados de primeira ordem para se tornar estacionária.

O PIB e a Taxa de Desemprego estão no estudo para medir a relação das ações com a atividade econômica, entretanto parece não haver uma relação significante entre essas variáveis e os investimentos em ações da construção civil, pelo menos não de modo contemporâneo no período em que o estudo foi feito. $\mathrm{O}$ mesmo vale para o CDI, talvez seu efeito não ocorra de forma concomitante, vindo a ser detectado somente em análises causais.

Além disso, deve-se atentar para a qualidade do ajuste do modelo medida pelo coeficiente $R^{2}$ ajustado das regressões. Observando a tabela, tem-se que os modelos se ajustaram em torno de 0,353 (BRML3) e 0,648 (MRVE3), com quatro equações explicando mais do que $50 \%$ da variabilidade das observações em relação a sua média.

\section{Reais}

\subsection{Modelo versus Retornos}

A melhor forma de visualizar se um modelo é ou não bem ajustado, além de observar suas estatísticas, é testando-o em dados futuros. Para ficar mais claro se o modelo é suficientemente adequado para que se possa tentar prever algo no mercado acionário, construiu-se a tabela 5, comparando os retornos projetados pelo modelo criado e os retornos reais das ações no primeiro semestre de 2012. Chamou-se de Modelo C os resultados referentes ao modelo e de Real, os retornos reais no período.

Sabe-se que um período de seis meses é considerado uma amostra muito curta para se chegar a uma conclusão estatisticamente confiável. Entretanto, analisando os modelos em relação aos retornos reais, percebe-se que esses não chegam a acertar os resultados, mas parecem captar os rumos que o mercado seguirá. Ressaltando novamente que um período de seis meses é pequeno para avaliar adequações dos modelos e que um bom ajuste no passado não garante acertos no futuro.

\section{CONSIDERAÇÕES FINAIS}

Inicialmente, foram feitos testes de raiz unitária para verificar a estacionariedade das séries, e aquelas que foram consideradas não estacionárias receberam tratamentos de diferenciação. Após isso, a análise de regressão multivariada foi utilizada e foram criados modelos relacionando os retornos das ações com os retornos dos indicadores. Essas equações se mostraram bem simples, com os maiores modelos possuindo no máximo cinco variáveis independentes. Analisando esses modelos, concluiu-se que o indicador de atividade Índice Bovespa foi o de maior relevância estatística entre todos os indicadores escolhidos, demonstrando ter uma relação contemporânea com o movimento de todas as ações do setor imobiliário deste estudo. Logo atrás do Ibovespa, vem a Taxa de Câmbio, que demonstrou poder explicativo em relação a maioria dos ativos do setor. A inflação se mostrou de pequena influência, afetando somente três ações, e tendo seu real efeito de forma dúbia, influenciando algumas ações positivamente e outras negativamente. Todos os outros indicadores não demonstraram impactar a forma como os investidores aplicam seus recursos no setor da construção civil, pelo menos não de modo contemporâneo.

Portanto, conclui-se que existe uma relação explicativa concomitante entre as ações do setor de construção civil brasileiro e alguns indicadores econômicos, sendo possível criar modelos que ajudam a explicar o modo como as ações se comportam frente a mudanças econômicas representadas por esses índices. 
Tabela 5: Comparação entre os modelos e dados reais

\begin{tabular}{|c|c|c|c|c|c|}
\hline \multicolumn{3}{|c|}{ BISA3 } & \multicolumn{3}{|c|}{ BRML3 } \\
\hline Mês & Modelo C & Real & Mês & Modelo C & Real \\
\hline janeiro-12 & $19,83 \%$ & $24,04 \%$ & janeiro-12 & $12,62 \%$ & $5,24 \%$ \\
\hline fevereiro-12 & $6,30 \%$ & $3,17 \%$ & fevereiro-12 & $5,72 \%$ & $13,10 \%$ \\
\hline marco-12 & $-9,16 \%$ & $-11,80 \%$ & marco-12 & $-0,71 \%$ & $5,17 \%$ \\
\hline abril-12 & $-9,39 \%$ & $-15,20 \%$ & abril-12 & $-2,94 \%$ & $3,14 \%$ \\
\hline maio-12 & $-21,79 \%$ & $-30,66 \%$ & maio-12 & $-10,76 \%$ & $-8,19 \%$ \\
\hline junho-12 & $-1,01 \%$ & $-7,28 \%$ & junho-12 & $1,01 \%$ & $6,48 \%$ \\
\hline & CYRE3 & & & GFSA3 & \\
\hline Mês & Modelo C & Real & Mês & Modelo C & Real \\
\hline janeiro-12 & $19,39 \%$ & $9,03 \%$ & janeiro-12 & $17,94 \%$ & $15,78 \%$ \\
\hline fevereiro-12 & $10,08 \%$ & $3,03 \%$ & fevereiro-12 & $6,15 \%$ & $6,46 \%$ \\
\hline marco-12 & $0,88 \%$ & $-11,31 \%$ & marco-12 & $-4,83 \%$ & $-11,52 \%$ \\
\hline abril-12 & $-15,43 \%$ & $-8,22 \%$ & abril-12 & $-8,65 \%$ & $-20,63 \%$ \\
\hline maio-12 & $-16,49 \%$ & $-3,57 \%$ & maio-12 & $-22,00 \%$ & $-29,16 \%$ \\
\hline junho-12 & $4,72 \%$ & $2,71 \%$ & junho-12 & $-1,89 \%$ & $3,95 \%$ \\
\hline & MRVE3 & & & PDGR3 & \\
\hline Mês & Modelo C & Real & Mês & Modelo C & Real \\
\hline janeiro-12 & $23,70 \%$ & $25,70 \%$ & janeiro-12 & $19,55 \%$ & $20,00 \%$ \\
\hline fevereiro-12 & $10,81 \%$ & $-1,98 \%$ & fevereiro-12 & $7,30 \%$ & $0,55 \%$ \\
\hline $\operatorname{marco} 0-12$ & $-8,49 \%$ & $-8,35 \%$ & $\operatorname{marco}-12$ & $-6,56 \%$ & $-16,42 \%$ \\
\hline abril-12 & $-9,27 \%$ & $-15,92 \%$ & abril-12 & $-6,94 \%$ & $-30,02 \%$ \\
\hline maio-12 & $-22,16 \%$ & $-25,67 \%$ & maio-12 & $-18,29 \%$ & $-28,66 \%$ \\
\hline junho-12 & $0,57 \%$ & $5,68 \%$ & junho-12 & $0,61 \%$ & $3,24 \%$ \\
\hline & RSID3 & & & & \\
\hline Mês & Modelo C & Real & & & \\
\hline janeiro-12 & $26,33 \%$ & $18,00 \%$ & & & \\
\hline fevereiro-12 & $9,01 \%$ & $4,15 \%$ & & & \\
\hline março-12 & $-17,36 \%$ & $-4,46 \%$ & & & \\
\hline abril-12 & $-4,28 \%$ & $-21,57 \%$ & & & \\
\hline maio-12 & $-32,77 \%$ & $-34,08 \%$ & & & \\
\hline junho-12 & $-7,02 \%$ & $-9,04 \%$ & & & \\
\hline
\end{tabular}

Fonte: Elaborado pelos autores

Sugere-se que trabalhos futuros possam realizar o mesmo tipo de estudo no setor de construção civil em bases com uma quantidade superior de dados, de modo que os retornos possam ser agrupados em trimestres, quadrimestres, semestres ou até anualmente, para verificar se as relações traçadas por este trabalho se mantêm. Seria interessante acrescentar variáveis fundamentalistas, os preços dos imóveis brasileiros e a oferta de crédito na economia nacional. Um trabalho analisando as relações causais dos indicadores para com as ações do setor avaliaria o grau preditivo dos indicadores com as ações. Sugere-se também que estudos parecidos sejam feitos em outros setores da economia brasileira, se possível mantendo o foco em ações em vez de índices de ações, que acabam por generalizar para todo um setor conclusões a respeito do seu agrupamento, algo que nem sempre é verdade.

\section{REFERÊNCIAS BIBLIOGRÁFICAS}

ADRANGI, B.; CHATRATH, A.; SANVICENTE, A. Z. Inflation, Output, And Stock Prices: Evidence From Brazil. The Journal of Applied Business Research, vol. 18, n. 1, 2002, p. 61-77. 
ASAOLU, T. O.; OGUNMUYIWA, M. S. An Econometric Analysis of the Impact of Macroeconomic Variables on Stock Market Movement in Nigeria. Asian Journal of Business Management, vol. 3, n. 1, fevereiro de 2010, p. 72-78.

BERGH, D. D.; GIBBONS, P. The Stock Market Reaction to the Hiring of Management Consultants: A Signalling Theory Approach. Journal of Management Studies, vol. 48, n. 3, may, 2011, p. 544-567.

BERTELLA, M. A.; SILVA, R.; PEREIRA, R. A. M. Cointegração e Causalidade entre Indicadores Macroeconômicos e Índice Bovespa. Apresentação em Seminário, 2009.

BOAVENTURA, R.; As variáveis fundamentalistas no apreçamento de ativos nos setores elétrico, siderúrgico e telecomunicações na bovespa. Dissertação de Mestrado, Rio de Janeiro, Universidade Estácio de Sá, 2009.

BONE, R. B. Existe causalidade entre as ações da Petrobrás Holding e o Ibovespa no período de 1994-2002?. $2^{\circ}$ Congresso Brasileiro de P\&D em Petróleo e Gás.

BOUCHER, C. Stock Prices, Inflation and Stock Returns Predicability. Revue de l'association française de finance, vol. 27, n. 2/2006, p 71-101.

BUSSAB, W. O.; MORETTIN, P. A. Estatística Básica. $5^{\circ}$ edição, Editora Saraiva, 2004.

CAMPBELL, J. Y.; VUOLTEENAHO, T. Inflation Illusion and Stock Prices. The American Economic Review, vol. 94, maio de 2004, p 19-23.

CASTRO, M. V. S. C. Uma análise empírica da relação entre inflação e retornos do mercado acionário brasileiro. Dissertação de Mestrado, Rio de Janeiro, Instituto Brasileiro de Mercado de Capitais, 2009.

DASGUPTA, A.; PRAT, A.; VERARDO, M. Institutional Trade Persistence and Long-Term Equity Returns. The Journal of Finance, vol. 66, n. 2, april, 2011, p. 635-653.

DEPARTAMENTO INTERSINDICAL DE ESTATÍSTICA E ESTUDOS SOCIOECONÔMICOS. Estudos e Pesquisas: Estudo Setorial da Construção. Em http://www.dieese.org.br/esp/estudos_set oriais.xml, n.56, abril de 2011.

DICKEY, D. A.; FULLER, W. A. Distribution of the Estimators for Autoregressive Time Series With a Unit Root. Journal of the American Statistical Association, vol. 74, n. 366, junho de 1979, p. 427-431.

EDGERTON, J. Agency Problems in Public Firms: Evidence from Corporate Jets in Leveraged Buyouts. Journal of Finance, Vol. LXVII, No. 6, pp. $2187-$ 2214, December 2012.

FAMA, E. F. Stock Returns, Real Activity, Inflation, and Money. The American Economic Review, vol. 71, n. 4, setembro de 1981, p. 545-565.

GESKE, R.; ROLL, R. The Fiscal and Monetary Linkage Between Stock Returns and Inflation. The Journal of Finance, vol. 38, n. 1, março de 1983, p 1-33.

GJERDE, Ø.; SÆTTEM, F. Causal relations among stock returns and macroeconomic variables in a small, open economy. Journal of International Financial Markets, Institutions and Money, vol. 9, n. 1, 1999, p 61-74.

GRÔPPO, G. S. Causalidade das variáveis macroeconômicas sobre o IBOVESPA. Dissertação de Mestrado, 
Piracicaba, Universidade de São Paulo, 2004.

GULTEKIN, B. Stock Market Returns and Inflation: Evidence from Other Countries. The Journal of Finance, vol. 38, n. 1, março de 1983, p. 49-65.

FERRI, F.; MABER, D. A. Say on Pay Votes and CEO Compensation: Evidence from the UK Review of Finance (2013) 17 (2): 527-563

HENDERSHOTT, T.; JONES, C. M.; MENKVELD, A. J. Does Algorithmic Trading Improve Liquidity ?. The Journal of Finance, vol. 66, n. 1, feb. 2011, p. 1-33.

HO, C. M.; Does Overconfidence Harm Individual Investors? An Empirical Analysis of the Taiwanese Market. Asia Pacific Journal of Financial Studies, vol. 40, 2011, p. 658-682.

MAHMOOD, W. M. W.; DINNIAH, N. $M$. Stock Returns and Macroeconomics Variables: Evidence from the SixAsian Pacific Countries. International Research Journal of Finance and Economics, n. 30, 2009, p. 154-164.

MARGARIDO, M. A.; ANEFALOS, L. C. Testes de raíz unitária e o software SAS. Agricultura em São Paulo, vol. 46, n. 2, 1999, p. 19-45.

MATOS, O. C. Desenvolvimento do Sistema Financeiro e Crescimento Econômico no Brasil: Evidências de Causalidade. Trabalhos para Discussão, Brasília, n. 49, setembro de 2002, p. 1-64.

RIBAS, J. R.; VIEIRA, P. R. C. Análise Multivariada com o uso do SPSS. $5^{\circ}$ edição, Rio de Janeiro, Editora Ciência Moderna, 2011.

SINGH, D. Causal Relationship Between Macro-Economic Variables and Stock Market: A Case Study for India. Pakistan Journal of Social
Sciences, vol. 30, n. 2, dezembro 2010, p. 263-274. 